\title{
La Gestion des Ressources Humaines, Precision Terminologique et Apercu Historiques -Une Revue de Litterature-
}

\author{
Hamid Latif, PhD in management sciences
}

ENCG, Hassan First University Settat, Morocco

Safaa Zakariya, PhD student

FSJES, Hassan First University Settat, Morocco

Fouad Elbiyaali, PhD in Economics

FSJES, Mohamed V University, Rabat, Morocco

Doi:10.19044/esj.2021.v17n35p178

Submitted: 03 September 2021

Accepted: 29 September 2021

Published: 31 October 2021
Copyright 2021 Author(s)

Under Creative Commons BY-NC-ND

4.0 OPEN ACCESS

Cite As:

Latif H., Zakariya S. \& ElbiyaaliF. (2021). La Gestion des Ressources Humaines, Precision Terminologique et Apercu Historiques -Une Revue de Litterature-. European Scientific Journal, ESJ, 17 (35), 178. https://doi.org/10.19044/esj.2021.v17n35p178

\section{Résumé}

En consultant les recherches de nombreux spécialistes en sciences de gestion, il a été constaté, au fil des années, une valorisation sans cesse grandissante pour les ressources humaines $(\mathrm{RH})$ en entreprise. Non seulement le rôle et l'importance de ces RH a changé, mais elles sont devenues des actifs stratégiques. Ce qui accorde, encore plus d'importance aux RH et à la GRH. Longtemps regardée comme une activité de support aux autres fonctions de l'entreprise, la GRH représente maintenant un outil stratégique qui permet à toute entreprise de se démarquer de ses concurrents. L'évolution des mentalités, le développement technologique ainsi que les erreurs du passé ont permis à la GRH de devenir un domaine extrêmement important dans les organisations. D'ailleurs, depuis quelques années, les pionniers de la discipline de GRH semblent de plus en plus s'accorder pour affirmer que la GRH constitue désormais un élément essentiel dans l'atteinte des principaux objectifs organisationnels. La GRH a intéressé, depuis quelques années, les débats scientifiques en sciences de gestion. Elle reste une discipline relativement jeune parmi les différentes branches de la gestion. Afin de comprendre davantage les théories et les pratiques actuelles concernant la 
GRH, il est intéressant de définir les principaux concepts et de se pencher sur les grandes phases de son histoire. Afin d'apporter une vision objective, claire et synthétique, on s'est appuyé sur sur une revue de la littérature structurée et approfondie et ce en consultant les principaux ouvrages, publications scientifiques, rapports, thèses et magazines spécialisés dans le domaine.

Mots clés: Gestion des Ressources Humaines, Pratique de Gestion des Ressources humaines, Gestion stratégique des Ressources Humaines

\title{
Human Resources Management : Terminological Precision and Historical Overview -Literature review-
}

\author{
Hamid Latif, PhD in management sciences \\ ENCG, Hassan First University Settat, Morocco \\ Safaa Zakariya, PhD student \\ FSJES, Hassan First University Settat, Morocco \\ Fouad Elbiyaali, PhD in Economics \\ FSJES, Mohamed V University, Rabat, Morocco
}

\begin{abstract}
By consulting the research of numerous specialists in management sciences, it has been observed, over the years, an ever-increasing value for human resources in companies. Not only has the role and importance of human resources changed, but they have become strategic assets. This gives even more importance to human resources and HRM. Long regarded as a support activity for other business functions, Human Resources Management (HRM) now represents a strategic tool that allows any business to stand out from its competitors. Changing mentalities, technological advancements as well as the mistakes of the past have allowed HRM to become an extremely important area in organizations. Moreover, in recent years, the pioneers of the discipline of HRM seem more and more to agree that HRM is now an essential element in achieving the main organizational objectives. For several years now, HRM has been of interest in scientific debates in management sciences. It remains a relatively young discipline among the different branches of management. In order to better understand the theories and current practices concerning HRM, it is interesting to define the main concepts and to look at the main phases of its history. In order to provide an objective, clear and synthetic vision, we relied on a structured and in-depth literature review by consulting the main
\end{abstract}


books, scientific publications, reports, theses and magazines specializing in the field.

Keywords: Human Resources Management (HRM), strategic human resources management, HRM practices

\section{Introduction}

En consultant les recherches de nombreux spécialistes en sciences de gestion, il a été constaté, au fil des années, une valorisation sans cesse grandissante pour les ressources humaines $(\mathrm{RH})$ en entreprise. Non seulement le rôle et l'importance de ces RH a changé, mais elles sont devenues des actifs stratégiques. Ce qui accorde, encore plus d'importance aux RH et à la GRH. Longtemps regardée comme une activité de support aux autres fonctions de l'entreprise, la GRH représente maintenant un outil stratégique qui permet à toute entreprise de se démarquer de ses concurrents. L'évolution des mentalités, le développement technologiques ainsi que les erreurs du passé ont permis à la GRH de devenir un domaine extrêmement important dans les organisations. D'ailleurs, depuis quelques années, les pionniers de la discipline de GRH semblent de plus en plus s'accorder pour affirmer que la GRH constitue désormais un élément essentiel dans l'atteinte des principaux objectifs organisationnels.

La GRH a intéressé, depuis quelques années, les débats scientifiques en sciences de gestion. Elle reste une discipline relativement jeune parmi les différentes branches de la gestion. Afin de comprendre davantage les théories et les pratiques actuelles concernant la GRH, il est intéressant de définir les principaux concepts et de se pencher sur les grandes phases de son histoire. Afin d'apporter une vision objective, claire et synthétique, on s'est appuyé sur sur une revue de la littérature structurée et aprofondie et ce en consultant les principaux ouvrages, publications scientifiques, rapports, thèses et magazines spécialisés dans le domaine.

\subsection{Définitions de la GRH}

La GRH est considérée comme un domaine de recherche vaste et souvent mal délimité (Garand, 1999). En effet et pour remédier à de probables et possibles ambiguïtés ou confusions, nous allons accorder, et d'une manière particulière, l'importance à sa définition. La GRH est une fonction de l'entreprise qui a pris ces dernières années une importance considérable en égard aux changements de l'environnement externe. Bien qu'elle semble bien installée parmi les activités essentielles de l'entreprise, sa définition reste une tâche délicate. Dans cette recherche, il n'est pas question de faire une liste ou d'inventorier toutes les définitions de la GRH, mais nous allons répertorier quelques-unes pour nous permettre de la mieux circonscrire. Comme l'indique 
Jackson et Schuler (1995), l'expression GRH est considérée comme un « terme ombrelle » utilisé selon différents sens et dans différents contextes. Pour Bournois et Brabet (1993), ce concept a été employé pour la première fois en 1817 par Springer, un économiste américain, pour révéler en termes comptables le coût de l'utilisation des hommes (Benchemam et Galindo 2015). La GRH c'est d'abord de la gestion, c'est-à-dire concevoir, conduire et contrôler un processus de décision (Le Gall, 2011). Selon le dictionnaire Larousse, le mot gestion signifie : action ou manière de gérer, d'administrer, de diriger, d'organiser. Ceci implique que toute entreprise doit déterminer une stratégie de gestion en intégrant un volet Ressource Humaine « $\mathrm{RH}$ » pour son développement et ce, quelle que soit sa taille. D'après (Marciano, 1995), le terme « RH » aurait été créé par Peter Ferdinand Drucker (1954) dans son livre intitulé « The practice of management ». Selon cet auteur, il existait trois types de management soit le management d'entreprise, de direction et celui du personnel et du travail. C'est, donc en étudiant le management du personnel qu'est apparue la notion de « RH ». L'association des concepts « Gestion » et « RH » ne va pas de soi et soulève des questions comme : Peut-on parler de « ressources » humaines au même titre que de ressources techniques, financières et matérielles ? Ces ressources peuvent-elles être « gérées » au même titre que l'on gère un portefeuille d'actions ?. Boyer (2006) cité par Ferar (2016) a répondu à cette question en considérant que le concept RH comporte un côté positif et un autre restrictif : «il a, certes, un côté positif : reconnaître que les hommes sont une ressource, c'est admettre qu'ils ont une valeur, un coût ou un prix. Mais c'est en même temps restrictif dans la mesure où on ne saurait mettre sur un même plan l'énergie, les matières premières, les machines et les hommes qui les produisent où les utilisent ». Le côté restrictif d'une GRH n'est plus adapté selon Boyer (2006) aux exigences d'aujourd'hui et de demain. En consultant la littérature spécialisée en GRH, plusieurs auteurs et chercheurs se sont intéressés au sujet afin de tenter de définir de façon claire ce qu'est la GRH. Boivin (1986) cité par Garand (1992), suggère que la GRH est « l'ensemble d'activités mises en œuvre par les entreprises, afin d'acquérir, de maintenir, de former et d'utiliser efficacement les individus exerçant ou susceptibles d'exercer un travail productif ; l'élément capital de cette définition est l'efficacité et la perspective envisagée est celle de l'organisation ». Dans leur dernière recherche, Bélanger et al (1988) ont formulé une définition associant plusieurs activités et pratiques, actuellement considérées dans les études GRH dans une entreprise : «La GRH est l'ensemble des activités d'acquisition, de développement et de conservation des RH, visant à fournir aux organisations de travail une main-d'œuvre productive, stable et satisfaite ».

Acquisition des RH : planification des effectifs, description des fonctions ou des emplois, recrutement, sélection et accueil ; 
Développement des RH : apport de connaissances et habiletés additionnelles permettant d'accroître la performance ou d'assumer de nouvelles responsabilités ;

Conservation des RH : rémunération, avantages sociaux, évaluation des emplois, structure salariale, négociation et administration des rapports collectifs du travail, discipline, ....

Marciano (1995), dans le portrait des origines de la GRH qu'il a tracé, a conclu qu'il y a trois grandes familles de définitions de la GRH, et ce selon son évolution (Aït Razouk, 2007) :

La première famille: La GRH est principalement une forme d'évitement des syndicats et une forme subtile de contrôle patronal. Pour ce courant, les travailleurs et l'entreprise ne peuvent avoir aucun intérêt en commun ;

La deuxième famille : La GRH est synonyme de « gestion du personnel », l'existance d'un ensemble de pratiques susceptibles de faire une meilleure utilisation des travailleurs ;

La troisième famille, soutenue et défendue par Drucker (1954) et Bakke (1958), la GRH est une fonction de gestion qui vise notamment à assurer la compréhension, la conservation, le développement, l'utilisation et l'intégration des RH dans leur milieu de travail. Elle positionne les employés comme une ressource organisationnelle qui a une valeur et non comme une dépense.

Une autre définition est celle proposée par Igalens et Roussel (1998). Pour ces auteurs, la GRH est « l'ensemble des activités qui visent à développer l'efficacité collective des personnes qui travaillent pour l'entreprise. L'efficacité étant la mesure dans laquelle les objectifs sont atteints, la GRH aura pour mission de conduire le développement des RH en vue de la réalisation des objectifs de l'entreprise. La GRH définit les stratégies et les moyens en RH, les modes de fonctionnement organisationnels et la logistique de soutien afin de développer les compétences nécessaires pour atteindre les objectifs de l'entreprise ». Dans sa définition Pigeyre (2006) stipule que « la GRH a pour but de fournir à l'entreprise les RH dont elle a besoin pour atteindre en temps voulu les objectifs qu'elle s'est fixé ». La GRH, d'un point de vue gestionnaire, vient pour s'intéresser à la façon et à la manière dont elle peut contribuer à la performance de l'entreprise. Comme le précise Galambaud (1994), dans l'expression GRH, c'est le mot gestion qui est le plus important... Cette notion de gestion renvoie en effet à une rationalisation de pratiques qui concernent les salariés (Pigeyre, 2006). Selon Jackson, Schuler et al. (2002), la GRH constitue « l'ensemble des activités qui visent la gestion des talents et des énergies des individus dans le but de contribuer à la réalisation de la mission, de la vision, de la stratégie et des objectifs organisationnels » (Comeau-Vallée, 2009). De leur côté, St-Onge et al. (2004), 
définissent la GRH comme « un ensemble variable de pratiques qui visent à aider l'organisation à résoudre avec efficacité, efficience et équité les problèmes associés à la présence de personnels au sein d'une entreprise ». Pour ces auteurs la GRH constitue une fonction de gestion au même titre que les autres fonctions de l'entreprise. D'après cette définition, pour étudier la GRH il faut pencher sur les pratiques mises en œuvre dans une entreprise. Quant à Martory, Crozet, et Solnik (2016) et Guillot-Soulez (2017), la GRH peut être définie comme «la gestion des hommes au travail dans des organisations ». Une autre définition est celle présentée par Cadin et al. (2012) : « la GRH est l'ensemble des activités qui permettent à une organisation de disposer des RH correspondant à ses besoins en quantité et qualité ». Le Louarn (2008) quant à lui, définit la GRH comme étant un ensemble de décisions qui dressent un contrôle sur les ressources afin d'en dégager des résultats. L'Organisation Internationale du Travail (OIT) a défini, quant à elle, la GRH comme : «Élément du management qui est chargé de donner ses avis d'une façon générale sur toutes les questions touchant au facteur humain dans l'entreprise et en particulier remplir certaines tâches d'ordre administratif relatives à l'emploi, aux conditions de travail et au bien-être du personnel de l'entreprise ». Dans une définition adoptée par le RHEP ${ }^{1}$, la GRH se veut « une discipline des sciences sociales consistant à créer et à mobiliser des savoirs variés utiles aux acteurs et nécessaires pour appréhender, comprendre, négocier et tenter de résoudre les problèmes liés à la régulation du travail dans les organisations » (Benchemam \& Galindo, 2015; Cadin \& Guérin, 2015) . Selon Le Louarn et Wils (2001), cité par Pottiez (2011) « la GRH est une tentative de contrôle de performance individuelle ou collective en vue de contribuer à l'efficacité organisationnelle ». Ils notent que la GRH se concrétise par la mise en place des $3 \mathrm{P}$ : politiques, processus et pratiques. Une politique est un énoncé général qui traduit l'intention de l'entreprise. Un processus est un ensemble d'activités reliées entre elles en vue d'atteindre un résultat concret. Une pratique est une manière de faire de l'entreprise. Pour Dietrich et Pigeyre (2011) la GRH est constituée de :

Pratiques de gestion ou les pratiques de GRH, qui sont une manière de faire de l'entreprise (Louarn et Wils 2001) ;

Règles et normes (outils de GRH, règles juridiques, conventions collectives...);

Différents acteurs (en interne : la direction, le service $\mathrm{RH}$, les managers, les représentants du personnel et en externe : les pouvoirs publics, les syndicats, les consultants) ;

Politiques de gestion (déterminées par les stratégies d'entreprise en fonction des contextes externe et interne).

${ }^{1}$ Groupe de réflexion épistémologique et prospective en GRH 
Pour Sekiou (2001), la GRH consiste en des mesures (politiques, procédures, etc.) et des activités (recrutement, etc.) impliquant des $\mathrm{RH}$ et visant à une efficacité et une performance.

\section{La GRH à travers le temps}

Dans notre revue de la littérature, nous avons constaté que les principaux chercheurs et auteurs en GRH sont d'accord et affirment que celleci a connu des évolutions très importantes. Elle a passé d'une logique de minimisation de l'impact de la GRH sur la performance, à une logique d'intégration des variables humaines pour améliorer les performances de l'entreprise (Cornet et al. 2015). Selon des auteurs comme Marciano (1995), Langbert et Friedman (2002), Savall et Zardet (2001) et Peretti (2017), l'intérêt accordé aux ressources humaines a considérablement évolué au fil des siècles, de sorte que l'on peut distinguer plusieurs périodes historiques quant à cette évolution.

\subsection{La période préindustrielle et industrielle}

Les professeurs américains Langbert et Friedman, (2002), dans leur rétrospective de l'histoire de la GRH, retournent jusqu'au 17ème et 18ème siècle dans une première étape qu'ils identifient comme la période préindustrielle. Dans cette période, même s'il existe certaines lois et règles sur le traitement des individus, les serviteurs et l'esclavage sont choses habituelles et les lois existantes sont couramment enfreintes et violées. Il s'agit d'une période où la liberté de l'employeur est illimitée et où les méthodes de production sont manuelles et artisanales. La plupart des produits ont été fabriqués dans les petites usines ou à la maison de l'employeur (à domicile) Hassan Ibrahim Ballott (2002). Les historiens mentionnent des manifestations de pratiques et des modes de gestion des hommes dès l'antiquité, à travers les règles d'organisation implicites ou explicites et les systèmes de sanctions et de récompenses érigés dans toutes les communautés (Bouchez J.P, 1999 ; cité par Benchemam et Galindo 2015). Regam Lynda (2018), ajoute que selon Jean-Pierre Bouchez (1999), ces pratiques et ces modes de la gestion du personnel sont très anciens et ce à travers les différentes civilisations qu'a connu l'humanité. Ces civilisations ont principalement orienté l'effort humain vers des objectifs considérés par elles comme importants dans la construction de leurs communautés. Durant cette période, la fonction RH n'existe pas, c'est le propriétaire qui assumait toutes les responsabilités à savoir, l'embauche, le licenciement et la rémunération...

Dans son ouvrage, Peretti (2017) stipule que la fonction RH n'existe plus à cette période et que les témoignages sont rares. C'est à travers quelques romans, qu'il est possible de découvrir des exemples de pratiques de GRH : Victor Hugo (Les Misérables), la comtesse de Ségur (La Fortune de Gaspard), Hector Malot (Sans famille), Émile Zola (Au Bonheur des dames). Entre 
autres, ils ont décrit des pratiques d'embauche, de licenciement, de formation, de promotion, d'œuvres sociales.

Avec la révolution industrielle, émergeant dans la deuxième moitié du 18ème siècle (1750), l'augmentation de la productivité et l'encouragemant de nouveaux standards de la qualité de vie ont été considérés comme les axes d'une nouvelle idéologie économiste. C'est la disparition de l'esclavage et la naissance des pratiques de gestion modernes (Langbert \& Friedman, 2002). L'introduction de la machinerie a accentué la distinction entre les employeurs (propriétaires) et les employés et entraîne des changements marqués dans les modèles sociaux et dans les conditions de travail (Jamrog \& Overholt, 2004). Cette révolution industrielle a entraîné le passage progressif d'un système de production artisanale et agricole, vers l'activité manufacturière et une production de masse (Wils, 1992). Selon Rouillard, (2004), les relations personnelles et paternalistes qui caractérisent ce modèle de production artisanale font place aux travailleurs salariés perçus par les employeurs comme une marchandise dont le coût doit être minimisé (Pozzebon et al. 2007).

Devant les conditions de travail qui leur sont imposées, les employés de même spécialisation se regroupent et s'organisent contre les abus des employeurs (Jamrog \& Overholt, 2004). Ceux-ci, ayant pris conscience des problèmes sociaux générés par l'industrialisation, les employeurs ont décidé d'offrir un soutien à leurs salariés pour régler leurs problèmes personnels. C'est à partir de ce moment que le poste de « secrétaire au bien-être » ou « secrétaire social » a connu son emergence. Son principal rôle était de gérer et contrôler l'ensemble des salariés, tout en décourageant la constitution des syndicats (Marciano, 1995 ; Jean-Pierre Bouchez (1999) cité par Regam Lynda 2018). Il apparait comme le premier service spécialisé en GRH. De son côté Jean Fombonne (2001), constate que la fonction Personnel est souvent exercée par les patrons eux-mêmes et parfois par un mandataire, soit le directeur de l'usine ou secrétaire, si l'effectif est important (Peretti 2017). Cette période, riche en changements et en évolutions, a connu la naissance de quelques écoles et courants de pensés qui ont impacté d'une manière très claire l'évolution de la GRH, en contribuant à tracer ses composantes dans les entreprises.

Malgré la différence de ces courants, l'essentiel du propos était de rechercher la manière de tirer le meilleur parti des ressources humaines de l'organisation.

\subsection{Après la révolution industrielle : les écoles et approches qui ont façonné la GRH}

L'évolution de la GRH est fortement attachée aux évolutions de l'environnement économique des organisations. Son développement se fait progressivement du 19ème siècle à nos jours. Tout au long de cette période, 
plusieurs courants et approches sont apparus en réponse à des caractéristiques de l'environnement tant interne qu'externe des organisations de travail (Petit, Bélanger, Benabou, Foucher et Bergeron, 1993 ; cité par Bernard 2009 ; (Gagnon \& Arcand, 2011; Pigeyre, 2006), influençant ainsi la GRH. Les historiens ont montré que les pratiques de GRH ont précédé la fonction RH. «Le 19ème siècle est celui de la fonction Personnel sans service Personnel, le 20ème siècle est celui de la fonction Personnel avec service du Personnel » (Fombonne, 2001) (Cité par Igalens 2006, Peretti 2017). La GRH a évolué en fonction des idées et théories managériales qui véhiculent une " bonne » manière de gérer le travail (Pigeyre, 2006). On retrouve dans la plupart des manuels de GRH, les cinq approches qui ont façonné la GRH (StOnge, Audet, Haine et Petit 2004, cité par Bernard (2009); Arcand (2006) :

\section{a) L'école classique ou l'approche scientifique ou techniciste}

Au 20ème siècle, avec l'émergence de la société industrielle (marquée par l'exode rurale, la présence d'une main d'œuvre nombreuse, essentiellement ouvrière, non spécialisée et peu performante), le modèle fondé sur la tradition et la coutume cède progressivement la place un modèle rationaliste, soutenu par l'essor des sciences et des techniques (Peretti 1998). Taylor, Fayol et Weber sont les principaux précurseurs et fondateurs de cette école. Leur but est la recherche d'une solution unique et universelle aux problèmes rencontrés par les entreprises dans la gestion de leurs activités et ce afin d'améliorer la productivité. Pour Frederick Winslow Taylor (1856-1915) dans son œuvre « The Principles of Scientific Management » a systématisé sa pensée, aujourd'hui connue sous le nom du taylorisme qui est associée au modèle mécaniste. Il associait les problèmes au facteur humain découlant de méthodes de production inadéquates. À cette époque, le recours aux principes de l'organisation scientifique du travail (OST) devait permettre de trouver une meilleure manière d'obtenir le meilleur rendement possible. La science fournissait les réponses écartant ainsi la contribution des employés. Cette étude donne lieu à la formulation de solutions optimales d'organisation, de parcellisation et de réalisation des tâches (One Best Way). À partir de cette orchestration, une sélection optimale du personnel est opérée selon leurs qualifications ou leurs capacités, pour que chacun trouve sa place dans l'organisation (the right man on the right place). Un système de récompense au rendement est introduit selon les quantités produites (a fair day's pay for a fair's day work) (Moulette et Roques, 2014). Les pratiques de gestion se réalignent et l'approche scientifique du travail de Taylor devient un modèle de référence pour la majorité des organisations (Moulette et Roques 2014 ; Benchemam et Galindo 2015). C'est avec les contributions du Taylor, le développement de la taille des organisations, le développement du droit social et la syndicalisation croissante etc., que les premiers postes traitants 
spécifiquement de la GRH se sont créés et que la fonction RH voit le jour (Peretti 2017, 1998 ; Jamrog et Overholt 2004). Et ce à partir du moment aussi où le chef d'entreprise n'est plus en état de gérer l'ensemble du personnel qui lui est alloué (Peretti 2017, 1998). Des auteurs comme Peretti (1998), voient que c'est le poste de secrétaire social qui a évolué pour devenir service Personnel et ce en 1912, la date référence de la naissance de la fonction RH. Son rôle se concentrait principalement sur l'analyse et la classification des postes, le développement, la formation et la sélection des employés (Jacoby, 1985). Fayol quant à lui, s'est principalement intéressé aux problèmes de direction de l'entreprise et a lancé les fondements de la théorie administrative (Cadin \& Guérin, 2015). Un fonctionnement optimal de l'entreprise est, selon lui, dicté par 14 principes : Division du travail, autorité, discipline, unité de commandement, unité de direction, clarté de la hiérarchie, stabilité du personnel, initiative, ordre, équité, système de rémunération équitable, centralisation, subordination des intérêts particuliers à l'intérêt général, union du personnel (Moulette \& Roques, 2014). Max Weber de son côté, est à l'origine de la définition des « idéaux type ». L'idéal type permettant de mettre en place l'organisation optimale est qualifié de système bureaucratique : la structure hiérarchique et les compétences de chaque emploi sont clairement définies, les rémunérations sont fixes et dépendent des responsabilités et du niveau hiérarchique, la discipline est stricte, la personne n'est pas propriétaire de son poste, des règles écrites anticipent toutes les situations (Moulette et Roques 2014, Cadin et Guérin 2015). Cette école de pensée regroupe des courants de pensée aux préoccupations différentes mais marqués par une même approche de l'organisation, à savoir la recherche de la rationalité. (Rationalité productive: Taylor; Rationalité administrative: Fayol ; Rationalité structurelle: Weber). L'objectif de cette école est de trouver et de définir des règles pour une gestion optimale de l'entreprise. L'OST et la bureaucratie sont deux notions particulièrement utilisées dans les travaux de cette école (Moulette \& Roques, 2014). Selon cette perspective, la fonction RH est limitée au niveau de règles ou des méthodes à mettre au point afin d'atteindre les résultats escomptés (St-onge et al. 2004 ; cite par Bernard (2009). Pour Taylor, il existe une seule et bonne manière de gérer le personnel. Elle doit être mécaniste, parce que : premièrement, les employés sont sélectionnés selon les compétences spécifiées et sont donc interchangeables pour pourvoir des postes précisément décrits ; deuxièmement, le seul pouvoir légitime émane du droit de propriété qui fixe les prérogatives et obligations y afférentes, dans le cadre d'une hiérarchie bien organisée ; troisièmement, le ressort de l'action (la motivation) réside dans un raisonnement calculateur des individus au service de leur seul intérêt personnel (Peretti, 2017). Pour accompagner l'OST, Taylor prescrit un certain nombre de pratiques de GRH : le recrutement, la formation et la rémunération (Benchemam et Galindo, 
2015). Pour ce courant de pensée, la dimension humaine s'efface devant l'optimisation de la production considérée comme finalité prioritaire. Avant le taylorisme, l'ouvrier est un homme qui détient un savoir faire sur la base duquel l'employeur le recrutait; et l'entreprise est considérée comme un groupe d'hommes. Mais avec le taylorisme, ce sont les spécialistes de la direction qui savent et l'entreprise devient une organisation dont le rouage est le poste de travail (Galambaud, 1994). Les travaux de Ford, influencés par les principes de l'OST de Taylor, ont contribué à leur tour à l'évolution de la fonction Personnel. Ils proposent un modèle opérationnel réalisant une production de masse grâce au travail à la chaîne. La disponibilité d'une main d'œuvre qualifiée et en grand nombre est l'une des nécessités de ce modèle. Cependant, dans une phase qui a connu une crise de main d'œuvre, les entreprises ont puisé largement les compagnes, la population féminine et les travailleurs immigrés, qui sont peu qualifiés (Peretti, 1998, 2017). Ce courant a été largement critiqué pour leur recherche utopique d'un modèle idéal et universel, leur vision considérée très mécaniste de l'homme, les liens avec l'environnement sont négligés, l'ignorance des interactions entre les individus et l'organisation, etc. Ce qui a conduit à l'émergence de nouvelles approches fondées sur la sociologie et la psychologie.

\section{b) L'école des relations humaines ou L'approche Psychologique (1927-1932)}

L'école des relations humaines est un mouvement intellectuel apparu dans les années 30 et qui s'est préoccupé de l'étude des organisations. Elle a pris naissance avec, d'une part les travaux d'E. Mayo, (principalement ses deux ouvrages : «The Human Problems of an Industrial Civilization », 1933 et «The Social Problems of an Industrial Civilization», 1945; qui comportaient les expérimentations menées à Hawthorne dans les ateliers de la Western Electric Company et d'autre part, les travaux de Kurt Lewin (Galambaud, 1994). Cette école a conduit à prendre le contre-pied de l'approche mécaniste de l'école classique (Pesqueux, 2015). La crise de 1929 a remis en cause les fondements et théories de l'école classique rationaliste, qui étaient appliqués à grande échelle (déshumanisation du travail qui a réduit l'homme à l'état de machine, salaire au rendement, cadences infernales...). Considérées comme des théories simples, l'échec de leur application (un absentéisme important et une montée des conflits sociaux durs...) a engendré l'emergence d'un second courant qui est celui des relations humaines (Cadin \& Guérin, 2015 ; Peretti, 2013). Ce dernier, met l'accent sur l'homme dans sa réflexion. Il lui rend sa dignité et donne au travail un sens plus humain. Le principe est de considérer les employés comme des valeurs importantes à l'organisation, et qui devraient être gérés par des pratiques de GRH afin de 
procurer un avantage stratégique compétitif aux entreprises (Kaufman, 2001 ; cité par Comeau-Vallée (2009).

L'étude des dimensions émotionnelles, affectives et relationnelles des situations de travail sont le fondement des modèles ou des modes de management préconisés par ce courant. Une des causes considérées importantes, par ce courant, des augmentations du rendement était le développement des relations entre chercheurs et ouvriers (individuellement ou en groupe), ce qui entraîne un changement de leur attitude envers leur travail (Peretti, 2013). L'importance du volet psychosociologique, l'analyse des besoins et des facteurs de motivation des salariés, le management démocratique et participatif, considérés comme contributions proncipales des chercheurs de ce courant, ont permis de faire évoluer la place et le rôle des RH dans l'entreprise (Moulette \& Roques, 2014 ; Peretti, 1998, 2017).C'est grâce à ce courant, que les RH ont une place importante dans le choix de l'avenir des organisations. Il a souligné l'impact du facteur humain sur la performance (Herzberg, 1957 ; McGregor, 1960 ; Trist, 1963). Ce courant avait appuyé le principe selon lequel l'amélioration des conditions de travail des employés était le justif pour améliorer la production. Leurs travaux, considérés plus humanistes, ont été soutenus au détriment des propos de la gestion scientifique du travail (Aït Razouk, 2007). L'expérience de Mayo «L'effet d'Hawthorne » qui stipule que tout changement de l'environnement de travail, que ce soit amélioration ou d'une dégradation, se traduit par une plus grande efficacité des ouvrières. Deux explications et deux éléments riches de sens pour la GRH sont ainsi soulignés (Benchemam \& Galindo, 2015): Le fait que les chercheurs s'intéressent à leur travail leur apporte une motivation pour être plus efficaces : importance des facteurs psychosociologiques ; Il existe des normes informelles de production au sein de chaque groupe, ce qui distingue le rôle des groupes pour la gestion des hommes.

À la fin des années 1950, grâce aux contributions des partisans de ce courant (Likert, MacGregor, Maslaw et Herzberg), la fonction Personnel s'est améliorée dans les organisations. Ils ont développé de nouvelles idées basées sur l'importance de la motivation, santé psychologique de l'ouvrier, la satisfaction des besoins du personnel, les communications, la participation des ouvriers dans la vie de l'organisation, les congés, la retraite, etc. (J. Fombonne, 2001) cité par Peretti (2017). Pendant cette période, la gestion du Personnel devient beaucoup plus qualitative, avec l'arrivée de psychosociologues à la tête des fonctions RH. C'est la grande époque de remise en cause progressive du modèle taylorien et domination des séminaires de motivation. Avec l'importance et souci de l'humain le nom « Direction du Personnel» change pour devenir «Direction des Relations Humaines», mettant l'accent sur l'importance du passage du quantitatif au qualitatif. A partir de ce moment, plusieurs pratiques de GRH furent implantées dans les entreprises afin de 
répondre à ces nouveaux besoins de développement et d'accomplissement des employés. Un changement qui a permis au poste de spécialiste du personnel de reprendre du galon par rapport à celui de spécialiste des relations industrielles (Pozzebon et al., 2007).

Malgré les contributions intéressantes et importantes de ce courant de pensée, il a été critiqué pour sa focalisation sur le côté moral au détriment du côté matériel. Cette focalisation a provoqué quelques effets négatifs sur la productivité et le rendement des organisations. Parce que la satisfaction des besoins illimités des employés est une finalité impossible à atteindre. Parfois la satisfaction de quelques besoins est en contradiction avec les buts et finalités de l'organisation. Durant cette période, les chercheurs de ce courant, se sont rattachés et intéressés aux comportements des individus, aux interrelations entre groupes et au phénomène global de l'organisation (Lawrence et Lorsch, 1967). En outre, ce courant a permis l'abandon progressif de méthodes de supervision axées sur l'intimidation et la peur de perdre son emploi par des méthodes plus positives orientées vers l'installation d'un climat de justice et de respect mutuel. (St-onge.S, Audet M., Haines, V., And Petit, A. 2004 ; cite par Bernard, 2009).

\section{c) L'approche institutionnelle, légale et politique :}

Identifiée comme approche des relations du travail, elle s'est développée suite au déséquilibre des pouvoirs entre les employeurs et les employés, puis à l'autoritarisme des gestionnaires et à la précarité économique que vivaient les employés. L'instauration par l'État de règles légales et les négociations collectives ont été privilégiées. Selon Peretti (2017), c'est après la première guerre mondiale que l'État commence à intervenir dans la vie économique, à travers la mise en place des lois et règlements organisant les relations au travail. Dont principalement, les lois relatives au congé hebdomadaire (1906), à la négociation collective et les heures du travail (1919), aux assurances sociales $(1928,1930)$ etc. Les entreprises prennent conscience de la nécessité d'entretenir de bonnes relations et de prévenir ou de gérer des conflits et ce, en réaction à la poussée d'un mouvement de syndicalisation. Les services du «bien-être » se transforment pour devenir « services du Personnel » avec les finalités d'administrer les droits des salariés et de régler les problèmes de rémunération, de sélection, de formation, d'évaluation et de communication avec les syndicats. Le développement et la croissance de la GRH se sont accompagnés par une complexité du droit social et des lois du travail, qui ont connu une accélération très intéressante avec l'adoption des lois sur, la création du comité d'entreprise, la sécurité sociale, les délégués du personnel, le SMIG, etc. Par conséquent, afin de s'adapter avec ces lois et règlements, les entreprises recrutent des juristes dans le poste de directeur du personnel. Peretti (2017), voit que, selon les exigences qu'a 
imposé cette période, les responsables du Personnel doivent se professionnaliser dans les différents domaines, comme : la formation, la rémunération, la qualification, la négociation collective, etc. C'est entre 1945 et 1965 que la création des associations pour le partage des informations et des expériences sur les questions de personnel a affirmé la fonction Personnel (Peretti, 2017). A ce moment, la GRH formait un éventail d'activités juxtaposées, sans référence à une vision d'ensemble. Selon ce courant de recherche, la GRH est considérée comme une activité isolée sans prise en compte d'une vision d'ensemble (St-Onge.S et al , 2004); cité par Bernard (2009).

\section{d) L'approche systémique}

Cette approche considère l'organisation comme un système ouvert, c'est-à-dire un ensemble d'éléments articulés en fonction d'un objectif commun et en relations dynamiques les uns avec les autres et avec l'environnement. Elle nécessite une étude et analyse des éléments composants l'environnement qui ont un impact sur la GRH, ainsi que sur les objectifs de l'organisation. En effet, la GRH est considérée comme un sous-système de l'entreprise. Elle est dans l'obligation de poursuivre des objectifs en adéquation avec ceux du système global (St-Onge Sylvie, Michel Audet, Victor Haines, André Petit-Releve, 2004); cité par Bernard 2009).

\section{e) L'approche stratégique}

Avec une évolution très intéressante de la réglementation (Enrichissement du code du travail), des mutations technologiques, un environnement incertain et invisible, une concurrence internationale, etc., les organisations doivent s'adapter à ces changements, ce qui a modifié profondément les enjeux de la GRH (Peretti, 2017). Selon ce dernier "C'est dans les années 1980, que la fonction Personnel se transforme en fonction RH. Le changement d'appellation est un changement de perspective et de pratiques. La conception traditionnelle du Personnel perçu comme une source de coût qu'il faut minimiser laisse place à la conception d'un Personnel considéré comme une source dont il faut optimiser l'utilisation ». Le service du personnel, géré par un « directeur du personnel », a progressivement laissé la place dans les entreprises, à partir des années 1980, à un « service ressources humaines », dirigé par un «directeur des ressources humaines » (DRH). Ce changement d'une «fonction Personnel» à une «fonction RH» trouve essentiellement sa source dans la crise économique, qui pousse les entreprises à revoir l'organisation peu productive du travail pour s'adapter à une économie ouverte et de plus en plus internationale (Guillot-Soulez, 2017b). De leur côté, Ait Razouk et Bayad (2010), stipulent que traditionnellement, la fonction RH s'est occupée de la gestion essentiellement administrative des RH. Ça 
principale mission et vision est de contrôler les RH pour minimiser leurs coûts (Huselid, Jackson et Schuler, 1997, Storey, 2001). Les partisans de la Gestion Stratégique des Ressources Humaines (GSRH) ne sont pas d'accord avec cette vision de la GRH (Becker et Huselid, 2006 ; Beer et al., 1984 ; Guérin et Wils, 2006). Pour eux, la ressource humaine est une ressource dans laquelle il faut investir en mobilisant un ensemble de pratiques de GRH afin d'améliorer la performance des entreprises (Beer et al., 1984 ; Pfeffer, 1998 ; Wright et McMahan, 1992). Dans le cadre de l'analyse du rôle stratégique de la GRH, la revue Human Resource Management a consacré un numéro spécial en 1997 pour déterminer les principaux défis pour la GRH dans le 21ème siècle. L'idée défendue et soutenue par l'ensemble des auteurs est que la GRH est en train de connaître une transformation allant du sens administratif vers le rôle stratégique (Peretti, 2017). De plus en plus de recherches empiriques viennent conforter la conviction selon laquelle la GRH est une source précieuse d'avantage compétitif durable sur le marché. D'ailleurs, c'est grâce à ces recherches que la GSRH se constitue et se renforce actuellement. L'examen de la littérature spécialisée en GRH montre une augmentation des recherches dans cette direction : -comprendre et surtout mesurer le rôle stratégique de la GRH. Selon Marciano (1995) et Schmidt et al. (2004), c'est aux années 80 qu'a été introduit le terme de " gestion stratégique des ressources humaines " (GSRH), pour le distinguer de celui de GRH traditionnelle ou gestion du personnel. Comme le soulignent bon nombre d'auteurs et chercheurs, le rôle stratégique de la GRH n'est pas le simple fait du hasard. Il est le résultat d'un mouvement de changements environnementaux (politiques, technologiques, sociologiques et économiques), qui ont un impact sur l'importance croissante donnée aux divers acteurs sociaux qui composent les organisations (Arcand et al, 2004a). Cette approche est caractérisée par l'intégration d'un ensemble d'activités choisies en fonction de l'obtention de résultats. Il s'agit là d'un processus de formulation et de mise en œuvre des moyens appropriés en vue d'atteindre les objectifs d'une organisation et de réaliser sa mission, dans un contexte marqué par une concurrence acharnée. Pour la GRH, cette approche renvoie en général à une préoccupation touchant l'harmonisation des pratiques de GRH dans les organisations qui sont gérées dans une approche stratégique (Peretti, 2017). L'objectif global de l'organisation est réalisé par un ensemble des activités incluant les RH (Petit et al. 1993 ; St-Onge et al (2004); cité par Bernard (2009); Arcand (2006). Aux années 2000, Selon Peretti (2017), la GRH s'affirme comme "partenaire d'affaires » à l'entreprise, en particulier dans une conjoncture devenue plus difficile. Lawler III et Boudreau (2009) confirment à travers leur étude que devenir un «partenaire stratégique » est un impératif ayant émergé lors des dix dernières années et que celui-ci constitue à présent un passage obligé pour toute fonction $\mathrm{RH}$ visant la performance. Son rôle est d'anticiper et de conseiller les dirigeants et les 
managers sur les solutions RH en réponse aux enjeux du business (Peretti 2017). La priorité est alors donnée au business en ajustant les ressources et compétences aux besoins de l'entreprise. Cependant, dans le contexte de la crise qu'ont connu les années 2000, la fonction $\mathrm{RH}$, comme partenaire d'affaires, a montré ses limites. Ce qui a permis, par conséquent, la revalorisation d'une fonction $\mathrm{RH}$ plus proche des salariés « $\mathrm{RH}$ de proximité », considérée comme une orientation centrée sur les personnes et le développement de leurs talents (Peretti 2017). Aux années 2010 et vu l'importance du capital humain dans la compétitivité des entreprises, la mission de la fonction RH s'est développée, et devient à la fois un partenaire d'affaires et un partenaire des hommes.

Aujourd'hui, la grande partie des managers imposent l'approche stratégique en ce qui concerne la GRH et soulignent l'exigence de considérer les employés comme une ressource stratégique et comme un moyen pour faire face à la concurrence (Lawler III \& Boudreau, 2009; Peretti, 2017).

\subsection{La GRH au Maroc, Bref apercu Historique}

Depuis une dizaine d'années, le Maroc vit au rythme de grandes mutations et profonds changements d'ordre politique, économique, social...etc. Ces mutations ont été accompagnées par une prise de connaissance progressive de la GRH et de son rôle. Au Maroc, comme presque pour tous les pays du Maghreb, la GRH ne peut être décrite sans se référer aux différents événements historiques vécus par ce pays: la décolonisation, marocanisation, privatisation, ouverture et mondialisation, etc. (Baayoud \& Zouanat, 2006 ; Dufour \& Golli, 2006 ; Matmati, 2005).

L'implantation des entreprises multinationales ainsi que l'œuvre normative de l'État, surtout dans le domaine du droit du travail, ont leur impact sur la GRH (Dufour \& Golli, 2006). L'histoire de la fonction RH au Maroc est récente, elle est apparue avec la colonisation et l'introduction du capitalisme au début du siècle dernier. Aujourd'hui, elle a atteint un degré égal aux autres fonctions centrales de l'organisation (Dufour \& Golli, 2006 ; Essaid Bellal, 2014).

Afin de bien comprendre la GRH actuelle, il est nécessaire de faire un bref aperçu sur son évolution. Ci-dessous les principales périodes, les plus citées, marquant l'évolution de la GRH au Maroc :

a) La période coloniale : l'émergence de la GRH au Maroc et plus globalement au Maghreb, date du début du siècle dernier, avec la colonisation (Dufour et Golli 2006 ; Matmati 2005 ; Baayoud et Zouanat 2006). D'après Dufour et Golli (2006), le Maroc, comme c'est le cas pour les autres pays du Maghreb (Tunisie, Algérie), était à l'époque soumis à une discrimination en matière de législation du travail, par ce qu'il était soumis à un droit d'exception « code du travail 
d'outre-mer » qui confirmait une certaine discrimination face au travail entre la métropole et les colonies. Baayoud et Zouanat (2006) quant à eux, évoquent quelques éléments et points ayant un impact sur l'évolution de la GRH, principalement l'opposition catégorique du patronat français à la mise en place d'une législation du travail. Ces auteurs précisent, que pendant les situations et périodes de forte demande, le chef d'entreprise faisait recours au caporal, comme institution intérimaire. Le caporal assure le recrutement et le contrôle de proximité, comme missions principales. Durant cette première phase, la GRH se caractérisait par trois aspects (Baayoud et Zouanat, 2006) :

- La flexibilité de la main-d'œuvre,

- L'opposition systématique du patronat français à la mise en place d'une législation du travail,

- Un mode de gestion du personnel différent selon qu'on a affaire à la population ouvrière autochtone ou européenne. «On pouvait dire que le milieu du travail était décomposé en trois catégories de personnes. Il y avait, à la base, des ouvriers marocains, il y avait des cadres et des dirigeants qui étaient composés exclusivement de Français ».

Pendant cette pérdiode, où les autorités françaises ont interdit toute création des syndicats marocains, les syndidats français des travailleurs mettaient la pression sur les autorités coloniales pour reconnaître un certains droits aux travailleurs autochtones (Baayoud \& Zouanat, 2006). En résumé, cette période est marquée par des conditions d'exploitation totale de la main d'œuvre (Discrimination, Absence de protection sociale, etc.) et l'absence d'une fonction dédiée à la GRH.

b) De l'indépendance aux années 90 : Au lendemain de l'indépendance, le droit syndical a été reconnu par la constitution marocaine ainsi que la législation du travail (Dufour et Golli 2006). Cependant, dans la réalité, l'action syndicale est appréciée, de différentes manières, selon les contextes dans lesquels elle se pratique et s'éxerce. Dans les entreprises qui ont été nationalisées ou marocanisées et dans les établissements publics constitués après l'indépendance, elle est largement tolérée et intégrée. Mais elle est rejetée et combattue au sein des autres structures. Les pratiques de GRH ressemblent, pendant cette phase, à celles qui dominaient pendant la phase coloniale, dont la pratique du contournement des contraintes légales qui est restée une donnée structurelle du mode de fonctionnement et de gestion de ces entreprises (Baayoud \& Zouanat, 2006).

Aux années 1950, les entreprises multinationales qui existent déjà continuent leur activité sans modification. Pour la grande partie des 
PME cédées aux nationaux, la transmission et la passation s'est faite sans préparation à la prise de responsabilités ni transfert des savoirs ni formation. C'est pour cette raison que la majorité de ces entreprises cédées ont un management archaïque et rudimentaire. Les rapports concernant le volet social étaient, par conséquent, marqués par une GRH obéissante à la hiérarchie avec une organisation totalement taylorienne ou anachronique et particulièrement par une absence de dialogue et de développement des compétences des salariés (Essaid Bellal 2014). De leur côté Baayoud et Zouanat (2006), estiment que l'action syndicale qui s'est développée d'une manière progressive au fil des années 60, a contribué à la structuration des pratiques de GRH. Ils ont constaté, dans cette période, une différence et une dualité dans les pratiques de GRH utilisées, et ce selon que l'entreprise est structurée et à l'abri des aléas du marché et de la concurrence ou que l'entreprise ne l'est pas. Dans le premier groupe (entreprises structurées ou entreprises providences), la GRH se caractérise par:

La formalisation du système d'administration du personnel, considéré et vu comme très contraignant pour les entreprises concernées. Le développement des services sociaux pour compléter ceux fournis par l'État dans le cadre d'une mutualisation globale : couverture médicale, retraites, vacances et loisirs, différents types d'aides...

Le maintien de "bon rapports avec les partenaires sociaux », en développant une culture de la négociation et du dialogue social. Pour le deuxième groupe, composé principalement des PME et de certains grands groupes familiaux (entreprises patrimoniales), la GRH n'a pas vraiment changé de celle qui prévalait durant la phase coloniale. La création de l'association d'appui aux entreprises, regroupant les professionnels du domaine RH "AGEF" en 1971, dont l'objectif est le partage des expériences, la diffusion des bonnes pratiques locales ou internationales, et la construction de liens avec des associations similaires dans le monde, a constitué un facteur de progrès important et intéressant pour la GRH au Maroc (Matmati, 2005). Cette association a contribué à donner de la visibilité à la GRH et aux différentes parties prenantes dans le processus de GRH au sein des entreprises. En résumé cette période est marquée par une domination du secteur public (marocanisation) et l'émergence du secteur privé ainsi que la création de l'AGEF.

c) Fin des années 90 au début de ce millénaire : Au cours des années 1990, le Maroc a connu de grands changements tels que la libéralisation et l'internationalisation de l'économie (Zone de libre-

\footnotetext{
${ }^{21}$ Association Nationale des gestionnaires et formateurs des ressources humaines « site web officiel :
} http://www.agef.org.ma/) 
échange avec l'Union Européenne, Privatisation, Pression de la concurrence ...). Tous ces changements ont ouvert de nouvelles perspectives à l'évolution de la GRH. Le rôle de celle-ci n'est pas spécialement pour exécuter les ordres et instructions venant d'en haut ; mais elle doit être aussi anticipative, réactive et experte dans la résolution des problèmes humains dans l'entreprise (Baayoud \& Zouanat, 2006). Durant cette période, le Maroc a été engagé dans un véritable mouvement de refondation sociale pour encourager les entreprises à reconsidérer et repenser leurs pratiques de GRH et les syndicats à adopter une attitude plus participative et moins conflictuelle (Dufour \& Golli, 2006). Avec la volonté du Maroc de mieux s'intégrer à l'économie modiale et au respect des droits de l'Homme, cette dynamique a donné plus d'imporatnce et de légitimité à la fonction RH. C'est dans cette période que cette fonction a commencé son véritable développement (Essaid Bellal, 2014). Les résultats des enquêtes réalisées par Essaid Bellal (Cabinet DIORH) sur la fonction $\mathrm{RH}$, font ressortir que les entreprises marocaines adoptent des grappes de pratiques proches de celles des entreprises européennes (Frimousse \& Peretti, 2005). D'après (Matmati, 2005), les missions et pratiques de la GRH ont connu une évolution rapide grâce d'une part, aux profonds changements et mutations qu'a connu le royaume ces dernières décennies, et d'autre part, par les contributions des universités, des écoles de gestion, des cabinets de conseils et d'audit et des associations professionnelles. Ce développement des pratiques de GRH, est considéré comme une évolution naturelle basée sur une GRH «importée» et imposée par le contexte économique international. Un contexte, avec la vitesse de son évolution, ne laisse aucune chance pour l'entreprise qui n'instaure pas dans son organisation, une GRH bâtie sur la performance. En résumé cette période est marquée par l'accélération de l'internationalisation de l'économie marocaine, la prise de conscience de la valeur du capital humain et par conséquent le développement de la fonction RH. À partir des années 2000, la GRH a connu un considérable développement et notamment dans les grandes entreprises (El Adraoui, 2015; Louart \& Scouarnec, 2005). Selon les résultats publiés d'une enquête réalisée par le cabinet Diorh en 2004, les responsables RH sont de plus en plus qualifiés, 66,3\% ont une formation universitaire et $56 \%$ ont une formation initiale en $\mathrm{RH}$. En ce qui concerne les pratiques de GRH, certaines entreprises nationales tentent de s'aligner aux pratiques des multinationales qui, en général, sont importées des maisons mères principalement sises en Europe. Avec la croissance, particulièrement avec l'accroissement du nombre d'employés, la fonction RH se formalise peu à peu et elle devient 
progressivement une réelle GRH effectuée par un spécialiste. De plus, par cette montée du formalisme, la fonction RH s'insère graduellement dans une vision stratégique. Au Maroc, selon les résultats d'une enquête réalisée en 2012 par le cabinet DIORH, les pratiques de GRH ont connu un développement important et on commence à sentir une prise en compte des RH comme variable clé dans la stratégie de l'entreprise, et non plus comme un facteur générateur de coût.

\section{Conclusion}

Pour conclure et à la lecture des différentes définitions, nous pouvons remarquer qu'il n'existe pas de consensus formel sur une définition unique et précise. C'est-à-dire qu'une définition universelle en ce qui concerne la GRH, n'existe pas. Antérieurement, l'époque où la RH était considérée comme une annexe comptable et comme une variable de production que l'on ajustait au gré des besoins, la fonction RH était un simple service du personnel, principalement en charge de la paie et des embauches, souvent rattaché aux affaires administratives et financières. A partir des années 1930, la GRH va progressivement acquérir ses lettres de noblesses, avec notamment les premiers travaux destinés à évaluer l'impact des relations humaines sur la productivité des RH. L'intégration de la dimension sociale et humaine dans l'entreprise et la prise de conscience de ses effets, datent en réalité du $\mathrm{XX}^{\circ}$ siècle seulement et plus particulièrement à compter des années 1960. Historiquement, elle est la conséquence de nombreuses revendications, de mouvements sociaux, d'une législation du travail qui va progressivement prendre en compte la santé et la sécurité des salariés, etc. Certes on ne peut exclure une part de gestion budgétaire notamment par la maîtrise du coût des emplois, de la formation, du turnover, mais la fonction RH ne peut pas limiter son champ d'intervention et d'investigations à ces seules notions comptables. Si ces dernières décennies ont permis de repositionner la RH dans l'entreprise et lui offrir la place qu'elle n'avait pas, elle est encore trop timorée et considérée par certaines organisations comme un mal nécessaire, non comme un levier de performance. Elle reste toujours le parent pauvre avec des managers qui privilégient davantage le commerce, la production, la finance. Rare sont les entreprises, qui intègrent les RH comme un levier stratégique à part entière. La littérature en sciences de gestion reconnaît la place et l'importance du capital humain dans l'acquisition d'un avantage concurrentiel durable et dans la création de valeur. C'est par le biais de la GSRH que les organisations peuvent réellement créer un puissant outil mis à leur disposition. 


\section{References:}

1. Aït Razouk, A. (2007). Gestion stratégique des ressources humaines : recherche théorique et empirique sur la durabilité de la relation entre stratégie RH et performance (PhD Thesis). Nancy 2.

2. Ait Razouk, A., \& Bayad, M. (2010). Pratiques mobilisatrices des ressources humaines et innovation des PME: existe-t-il un lien? Colloque GRH, 1 Er Avril 2010, Montpellier, FRANCE.

3. Arcand, G. (2006). Étude du rôle de la culture nationale dans la relation entre les pratiques de GRH et de la performance organisationnelle: le cas des banques de vingt-deux pays d'Amérique du Nord, d'Europe et d'Asie (PhD Thesis). Metz.

4. Arcand, M. (2001). L'effet des pratiques de gestion des ressources humaines sur l'efficacité des caisses populaires Desjardins du Québec (PhD Thesis). Metz.

5. Arcand, M., Arcand, G., Bayad, M., \& Fabi, B. (2004a). Gestion stratégique des ressources humaines et performance de la firme: une validation de l'approche de la contingence. Revue Internationale Des Relations de Travail, 2(2), 14-25.

6. Arcand, M., Bayad, M., \& Fabi, B. (2002). L'effet des pratiques de gestion des ressources humaines sur l'efficacité organisationnelle des coopératives financières canadiennes. Annals of Public and Cooperative Economics, 73(2), 215-240.

7. Audet, M., Bélanger, L., Long, R. J., \& Galambaud, B. (1994). La gestion des ressources humaines: du modèle traditionnel au modèle renouvelé. Relations Industrielles/Industrial Relations, 49(1), 168187.

8. Baayoud, M., \& Zouanat, M. (2006). La GRH au Maroc: histoire et perspectives de reconfiguration. Ressources Humaines en Euroméditerranée, 2, 122-131.

9. Barney, J. B., \& Wright, P. M. (1998). On becoming a strategic partner: The role of human resources in gaining competitive advantage. Human Resource Management (1986-1998), 37(1), 31.

10. Becker, B. E., \& Huselid, M. A. (2006). Strategic human resources management: where do we go from here? Journal of Management, 32(6), 898-925.

11. Benchemam, F., \& Galindo, G. (2015). Mémentos LMD-Gestion des ressources humaines. Gualino éditeur.

12. Bernard, M. (2009). L'effet des pratiques de ressources humaines sur la performance sociale des employés dans un contexte de culture nationale ( $\mathrm{PhD}$ Thesis). Atelier national de reproduction des thèses. 
13. Bouamama, M. (2015). Nouveaux défis du système de mesure de la performance: cas des tableaux de bord ( $\mathrm{PhD}$ Thesis). Université de Bordeaux.

14. Boyer, L., \& Equilbey, N. (2003). GRH: nouvelles pratiques. Eyrolles, Paris.

15. Cadin, L., \& Guérin, F. (2015). La gestion des ressources humaines (4e éd). Dunod.

16. Chênevert, D., Filiatrault, È.-M., Lepine, I., \& Morin, D. (2008). Les rôles joués par les services de ressources humaines et la performance organisationnelle: $y$-a-t'il un lien?

17. Comeau-Vallée, M. (2009). La formalisation des pratiques de gestion des ressources humaines dans les entreprises d'économie sociale: une menace aux valeurs du secteur?

18. Des Horts, C.-H. B. (2004). La fonction RH, une fonction stratégique : discours ou réalité?

19. Dietrich, A., \& Pigeyre, F. (2011). La gestion des ressources humaines. La découverte Paris.

20. Dufour, L., \& Golli, A. (2006). Vers une convergence « contextualisée » des pratiques RH dans l'espace Euroméditerranéen. Pratiques d'audit social et de RSE dans la diversité de leurs environnements, 127-142. Dakar, Sénégal: IAS.

21. Dupont, C. (2013). Du discours stratégique à la construction de la fonction RH par les praticiens. XXII Conférence Internationale de Management Stratégique.

22. El Adraoui, H. (2015). Effets de la GRH sur la performance: validation du modèle configurationnel auprès d'un échantillon de 92 entreprises au Maroc. Question (s) de Management, (3), 43-56.

23. Essaid Bellal. (2014). L'évolution De La Fonction RH Au Maroc. Ressources humaines et responsabilités sociétales: Mélanges en l'honneur du Professeur Jean-Marie Peretti. EMS Editions.

24. Ferar, D. (2016). Construire des pratiques de management des ressources humaines durable au Maroc (L'Harmattan). Retrieved from

http://uh1.scholarvox.com/catalog/search/searchterm/Ferar,\%20Driss ?searchtype $=$ author

25. Frimousse, S., \& Peretti, J.-M. (2005). Apprentissage stratégique des pratiques de GRH, internationalisation des firmes et espace euromaghrébin. Management \& Avenir, (3), 45-61.

26. Gagnon, O., \& Arcand, G. (2012). L'augmentation de la performance organisationnelle par l'application de pratiques de GRH alignées à la stratégie d'affaires. AGRH. 
27. Galambaud, B. (1994). Une nouvelle configuration humaine de l'entreprise: le social désemparé. Paris: ESF.

28. Garand, D. J. (1992). Les pratiques de gestion des ressources humaines $(G R H)$ en petites et moyennes entreprises (PME): une synthèse de la documentation empirique ( $\mathrm{PhD}$ Thesis). Université du Québec à Trois-Rivières.

29. Grisé, J. (1997). Les ressources humaines en tant que source d'avantage concurrentiel durable. Québec: Faculté des sciences de l'administration de l'Université Laval, Direction de la recherche.

30. Guillot-Soulez, C. (2017a). Gestion des ressources humaines: 440 mots clés définis et expliqués Ed. 3.

31. Guillot-Soulez, C. (2017b). La gestion des ressources humaines. Gualino.

32. hassan Ibrahim Ballot, ب. ح. إ. (2002). إدارة الموارد البشرية من منظور البهية (1st) إستر اتيجي (1st ed., Vol. 1). دار النهضة العربية النظية

33. Iacono, G. (2008). Gestion des Ressources Humaines : Cinq défis pour l'avenir $\quad$ Ed. $\quad 2 . \quad$ Retrieved from https://international.scholarvox.com/catalog/book/10216410

34. Igalens, J. (2006). La GRH: principes, pratiques et critiques. Management et Gestion des Ressources Humaines: Stratégies, Acteurs et Pratiques, 25.

35. Jamrog, J. J., \& Overholt, M. H. (2004). Building a strategic HR function: Continuing the evolution. Human Resource Planning, 27(1).

36. Langbert, M., \& Friedman, H. (2002). Continuous improvement in the history of human resource management. Management Decision, 40(8), 782-787.

37. Le Gall, J.-M. (2011). La gestion des ressources humaines: "Que sais$j e$ ?» $\mathrm{n}^{\circ} 2646$. Presses universitaires de France.

38. Le Louarn, J. Y., \& Gosselin, A. (2000). GRH et profits: y a-t-il un lien? Effectif, Avril-Mai, 18-23.

39. Le Louarn, J.-Y. (2008). Les tableaux de bord ressources humaines: le pilotage de la fonction $R H$. Wolters Kluwer France.

40. Le Louarn, J.-Y., \& Wils, T. (2001). L'évaluation de la gestion des ressources humaines. Liaisons.

41. Lethielleux, L. (2018). L'essentiel de la Gestion des ressources humaines $\quad$ Ed. $12 . \quad$ Retrieved from https://international.scholarvox.com/catalog/book/docid/88861606?se archterm=Gestion\%20des\%20ressources\%20humaines

42. Louart, P., \& Scouarnec, A. (2005). Penser la GRH en Algérie, au Maroc et en Tunisie: de l'état des lieux aux perspectives de recherches et d'actions. Perspectives Sur La GRH Au Maghreb: Algérie-MarocTunisie. Coordonné Par Zahir Yanat et Aline Scouarnec, Vuibert. 
43. Marciano, V. M. (1995). The origins and development of human resource management. Academy of Management Proceedings, 1995, 223-227. Academy of Management Briarcliff Manor, NY 10510.

44. Matmati, M. (2005). La gestion des ressources humaines au Maghreb. YANAT Z., SCOUARNEC A.(Coor.)(2005), Perspectives Sur La GRH Au Maghreb: Algérie-Maroc-Tunisie, Chapitre, 16.

45. Moulette, P., \& Roques, O. (2014). Maxi Fiches de Gestion des ressources humaines (2e édition). Dunod.

46. Nicolas, E. (2014). Gestion des ressources humaines. Retrieved from https://international.scholarvox.com/catalog/book/docid/88831256?se archterm=Gestion\%20des\%20ressources\%20humaines

47. Peretti, J.-M. (1998). Ressources humaines et gestion du personnel. Vuibert.

48. Peretti, J.-M. (2013). Ressources humaines (Ed 14). Retrieved from http://www.scholarvox.com/reader/docid/88816327/page/1

49. Peretti, J.-M. (2017). Ressources humaines (Ed 16). Retrieved from http://uh1.scholarvox.com/catalog/book/docid/88848956?searchterm $=$ ressources $\% 20$ humaines $\% 2016 \mathrm{e} \% 20 \% \mathrm{C} 3 \%$ A 9 dition

50. Peretti, J.-M. (2018). Gestion des ressources humaines. Vuibert.

51. Pigeyre, F. (2006). Les modèles d'analyse de la GRH. Management et Gestion Des Ressources Humaines: Stratégies, Acteurs et Pratiques, 7.

52. Pozzebon, S., Coiquaud, U., Gosselin, A., \& Chênevert, D. (2007). La gestion des ressources humaines: d'hier à demain. Gestion, Vol. 32(3), 99-109. Retrieved from https://www.cairn.info/revue-gestion2007-3-page-99.htm

53. Regam Lynda, ر. ل. (2018). دور إدارة الموارد البشرية في تسيير التغبير في في ولائة المؤسسات الإقتصادية الكبرى في ولاية سطيف فئسئ (PhD Thesis).

54. Schmidt, G., Guéry, L., Mercier, E., \& Mottay, D. (2004). La fonction Ressources Humaines, levier de création de valeur? Du volontarisme des discours au scepticisme des acteurs. Congrès AGRH, 591-610.

55. Schuler, R. S., \& Tarique, I. (2007). International human resource management: a North American perspective, a thematic update and suggestions for future research. The International Journal of Human Resource Management, 18(5), 717-744.

56. Sekiou, L. (2001). Gestion des ressources humaines. De Boeck Supérieur.

57. Simen, Serge Francis. (2017). Pratiques de GRH dans les très petites entreprises sénégalaises : pertinence d'une gestion qui concilie tradition et modernité. XXVIe Conférence Internationale de Management Stratégique. 
58. Stewart, G. L., \& Brown, K. G. (2019). Human Resource Management. John Wiley \& Sons.

59. St-Onge, S., Audet, M., Haines, V., \& Petit, A. (2004). Faire de la GRH une valeur ajoutée. Gaëtan Morin Editeur, 659-687.

60. Thamain, J. L. (2009). Anticiper en GRH, prévision et prospective, un diagnostic des pratiques et des représentations. Management \& Avenir, (5), 272-289.

61. Tremblay, M., Chênevert, D., Simard, G., Lapalme, M.-È., \& Doucet, O. (2005). Agir sur les leviers organisationnels pour mobiliser le personnel: le rôle de la vision, du leadership, des pratiques de GRH et de l'organisation du travail. Gestion, 30(2), 69-78.

62. Trépé, A., Aubert, P., Bermond, F., Carrère, J.-M., \& Delanoue, M. (2010). L'évaluation de la performance de la fonction $R H$ : De la théorie à la pratique des DG. Mémoire d'expertise en vue de l'obtention d'un MBA en management des ressources humaines, Université de Paris Dauphine.

63. Trudel, J. M. (2011). Des pratiques de grh favorisant la contribution des employés aux besoins de flexibilité des organisations. Revue International Sur Le Travail et La Société, 9(20), 24-45.

64. Ulrich, D. (1996). Human Resource Champions: The Next Agenda for Adding Value and Delivering Results. Harvard Business Press.

65. Wils, T. (1992). La gestion des ressources humaines: du modèle traditionnel au modèle renouvelé. Montréal: Presses de l'Université de Montréal.

66. Wright, P. M., \& McMahan, G. C. (2011). Exploring human capital: putting 'human'back into strategic human resource management. Human Resource Management Journal, 21(2), 93-104. 